\title{
Picometer stable scan mechanism for gravitational wave detection in space
}

\author{
N. Rijnveld ${ }^{\mathrm{a}}$, J.A.C.M. Pijnenburga, \\ ${ }^{a}$ Dept. Space \& Science, TNO Science \& Industry, Stieltjesweg 1, 2628 CK Delft, The Netherlands
}

\begin{abstract}
Detection and observation of gravitational waves requires extremely accurate displacement measurement in the frequency range $0.03 \mathrm{mHz}$ to $1 \mathrm{~Hz}$. The Laser Interferometer Space Antenna (LISA) mission will attain this by creating a giant interferometer in space, based on free floating proof masses in three spacecrafts. Due to orbit evolution and time delay in the interferometer arms, the direction of transmitted light changes. To solve this problem, a picometer stable Point-Ahead Angle Mechanism (PAAM) was designed, realized and successfully tested. The PAAM concept is based on a rotatable mirror. The critical requirements are the contribution to the optical path length (less than $1.4 \mathrm{pm} / \mathrm{rt} \mathrm{Hz}$ ) and the angular jitter (less than $8 \mathrm{nrad} / \mathrm{rt} \mathrm{Hz}$ ). Extreme dimensional stability is achieved by manufacturing a monolithical Haberland hinge mechanism out of $\mathrm{Ti}_{6} \mathrm{Al}_{4} \mathrm{~V}$, through high precision wire erosion. Extreme thermal stability is realized by placing the thermal center on the surface of the mirror. Because of piezo actuator noise and leakage, the PAAM has to be controlled in closed-loop. To meet the requirements in the low frequencies, an active target capacitance-to-digital converter is used. Interferometric measurements with a triangular resonant cavity in vacuum proved that the PAAM meets the requirements.
\end{abstract}

Keywords: picometer, stability, scan, LISA, PAAM

\section{INTRODUCTION}

The picometer stable scan mechanism is a crucial element in the Laser Interferometer Space Antenna (LISA) mission ${ }^{1}$. The objective of the LISA mission is to observe and measure gravitational waves. Because of the extreme stability and low disturbance requirements, the detector will be created in space. The detector will consist of a giant interferometer with three measurement arms, travelling between three spacecraft each at a distance of 5 million kilometers from each other. Each spacecraft holds two free-floating proof masses, which provide the absolute reference for the interferometer arms. Because the disturbances acting on the proof masses are absolutely minimized, any path length changes measured by the interferometer arms can be attributed to gravitational waves.

Due to the evolution of the orbit during its trip around the sun, the laser beam angles have to be corrected for constantly. Not only the in-plane angles are affected, but also the so-called point-ahead angles, which correct for the offset caused by the time delay of the travelling light. The Point-Ahead Angle Mechanism (PAAM) is designed to perform the task of correcting the point-ahead angle.

The PAAM is developed and tested by TNO Science \& Industry, with the help of the Albert-Einstein-Institute in Hannover. As an Elegant Bread-Board (EBB), it has successfully gone through all performance and environmental testing and is ready to be integrated in a functional breadboard of the Optical Bench for a LISA spacecraft.

\section{DRIVING REQUIREMENTS FOR THE PAAM}

The PAAM is required to steer the incoming laser beam through a range of $\pm 824 \mu \mathrm{rad}$, while contributing minimally to the optical path delay (OPD) and contributing minimally to the angular jitter of the laser beam angle. During operation, the mechanism will follow an annual trajectory that runs through the entire range twice.

Optical and Infrared Interferometry II, edited by William C. Danchi, Françoise Delplancke, Jayadev K. Rajagopal, Proc. of SPIE Vol. $7734,77341 \mathrm{R} \cdot$ (C) 2010 SPIE · CCC code: 0277-786X/10/\$18 · doi: 10.1117/12.857040 


\subsection{Performance requirements}

Both the specification for the optical path delay (OPD) and the angular jitter are described using a noise shape function, as shown Equation 1.

$$
n(f)=\sqrt{\left(1+\frac{2.8 m H z}{f}\right)^{4}}
$$

The requirements for OPD and angular jitter, defined in terms of amplitude spectral density (ASD), are multiplied with this noise shape function. It is designed such that the requirements are relieved below $2.8 \mathrm{mHz}$, and constant above this frequency. The requirements only apply within the LISA measurement band width, which is defined to be between 0.03 $\mathrm{mHz}$ and $1 \mathrm{~Hz}$. Table 1 shows the requirements for OPD and angular jitter.

Table 1: Performance requirements for the PAAM.

\section{PERFORMANCE REQUIREMENTS}

\begin{tabular}{|ccc|} 
Description & Requirement & Unit \\
\hline Optical Path Delay & $1.4 \cdot n(f)$ & $\mathrm{pm} / \sqrt{\mathrm{Hz}}$ \\
Angular jitter & $16 \cdot n(f)$ & $\mathrm{nrad} / \sqrt{\mathrm{Hz}}$ \\
\hline
\end{tabular}

\subsection{Environmental requirements}

Several additional requirements make the design of the PAAM quite challenging. First of all, due to the sensitivity of the measurement set-up on the Optical Bench, no magnetic materials are allowed. This rules out the use of any electromagnetic actuators or bearings. Second, due to the strict requirements on stray light, the contamination requirements on the Optical Bench are extremely strict. Any outgassing materials, as well as mechanisms containing moveable parts with frictional contacts are to be avoided. Third, the mechanism will have to survive launch loads of $25 \mathrm{~g}$ RMS, without the use of a launch locking mechanism.

\section{POINT-AHEAD ANGLE MECHANISM DESIGN DESCRIPTION}

The PAAM consists of a mirror on a flexible rotational hinge, which can be actuated individually by either one of two piezo stacks. The angle is measured by a capacitive sensor. The system operates in closed loop. The overall design is shown in Figure 1, and its realization is shown in Figure 2. In the paragraphs below, the optical, mechanical and electrical design is described in detail. 


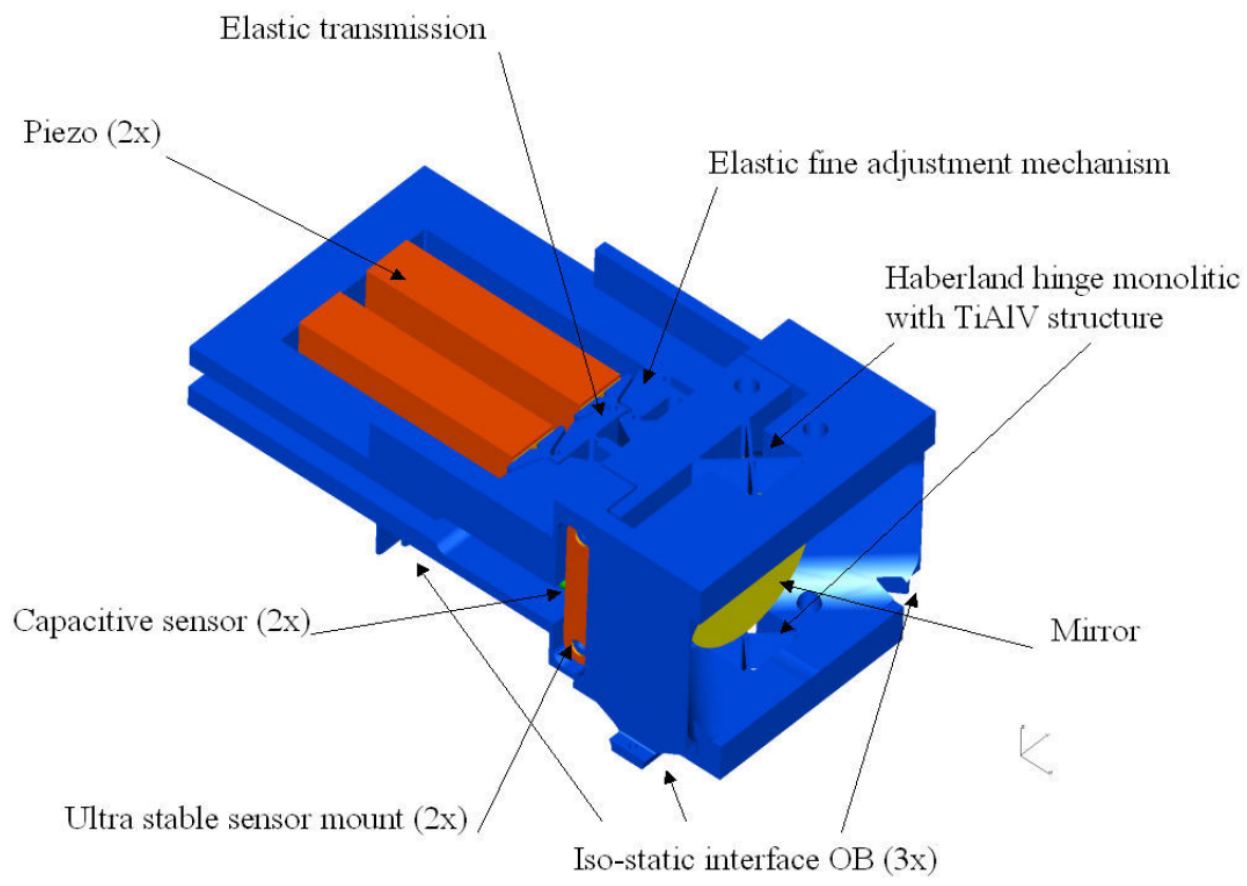

Figure 1: CAD design of the Point-Ahead Angle Mechanism as designed by TNO Science \& Industry, including all its components.

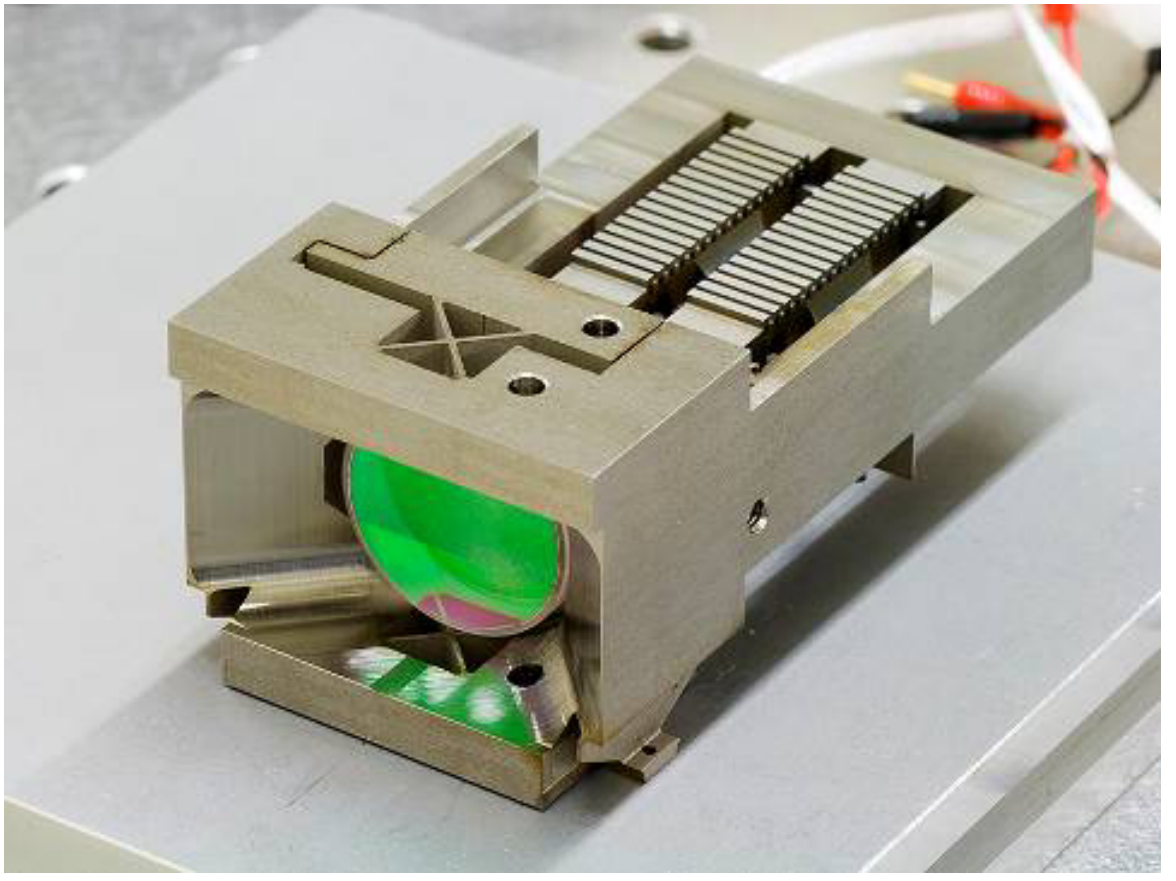

Figure 2: Realization of the Point-Ahead Angle Mechanism. The mirror is glued to a monolithical structure featuring a Haberland hinge. The mirrors can be actuated by one of two piezo-stacks. 


\subsection{Optical design}

The optical concept for the PAAM is a mirror rotating around an axis in the mirror plane. This concept is chosen over alternatives due to its low transmission losses and low complexity. The mirror is coated for the wavelength of $1064 \mathrm{~nm}$ and for the incoming angle of $45^{\circ}$. The reflectivity is specified as more than $99.9 \%$, and its flatness is better than $12 \mathrm{~nm}$ RMS over its entire diameter of $19.05 \mathrm{~mm}$.

The Optical Path Delay (OPD) has been identified as the most critical requirement. If the laser beam and the reflecting surface are perfectly aligned with the rotation, the OPD due to rotation is theoretically zero. Figure 3 schematically shows the optical path delay due to a rotation of the mirror, when the beam and surface are not perfectly aligned.

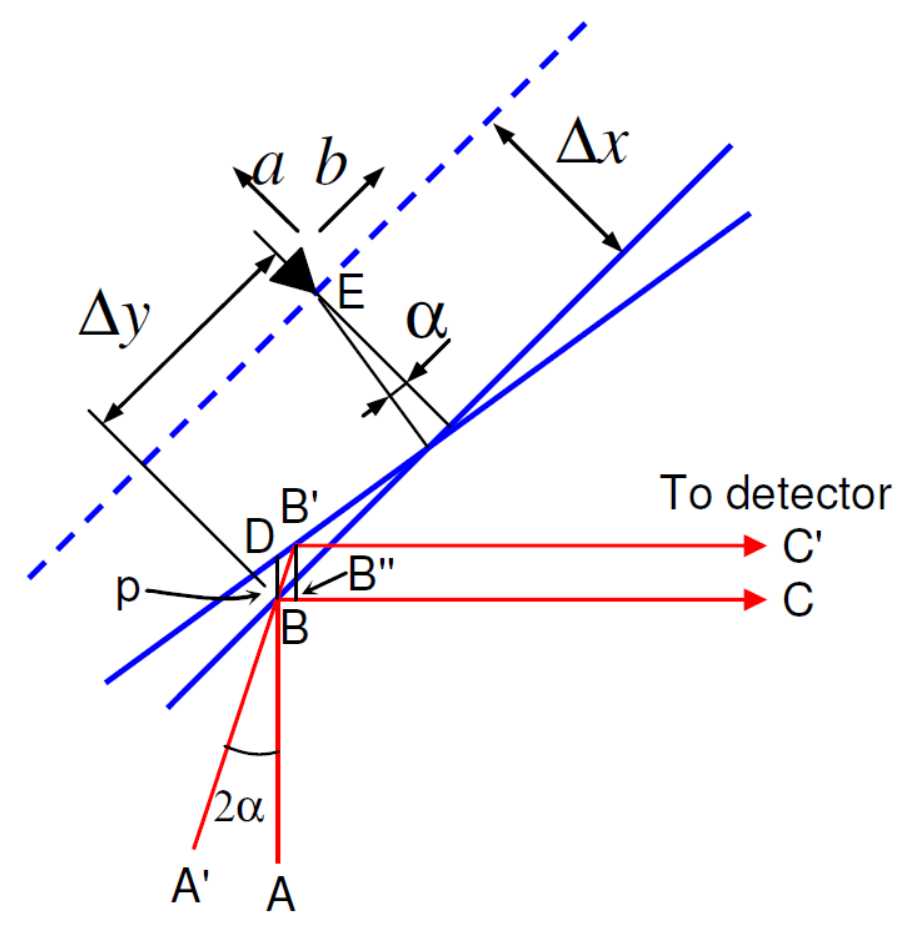

Figure 3: Schematic representation of the influence of alignment and jitter parameters of the mirror on the optical path delay of the incoming laser beam.

From Figure 3 it can be seen that the OPD equals the distance BB'-BB". For small angles, this can be approximated by $\mathrm{BD}$. The sensitivity of the distance $\mathrm{BD}$, and hence the OPD, to the most relevant parameters is listed in

Table 2. These parameters are the angular jitter, caused by rotation of the mirror around the optical axis in combination with an alignment offset, the longitudinal jitter, caused by piston sensitivity and angular jitter, and the rotation axis longitudinal jitter, caused by parasitical actuation forces through the Haberland hinge. Other misalignments of the optical axis and cross-couplings were shown to have negligible contribution to the Optical Path Delay. 
Table 2: Sensitivity of the OPD to relevant parameters:

\begin{tabular}{|c|c|c|c|c|}
\hline \multirow[b]{2}{*}{ Parameter } & \multicolumn{3}{|c|}{ SENSITIVITY OF THE OPD TO RELEVANT PARAMETERS } & \multirow{2}{*}{$\begin{array}{l}\text { Effect on OPD } \\
p m / \sqrt{H z}\end{array}$} \\
\hline & Description & Budget & Relation & \\
\hline$\Delta \mathrm{x}$ & $\begin{array}{l}\text { Static longitudinal } \\
\text { misalignment }\end{array}$ & $\pm 1000 \mu \mathrm{m}$ & - & 0 \\
\hline$\Delta y$ & $\begin{array}{c}\text { Static lateral } \\
\text { misalignment }\end{array}$ & $\pm 50 \mu \mathrm{m}$ & - & 0 \\
\hline$\Delta \alpha$ & Angular jitter & $8 \cdot n(s) n r a d / \sqrt{H z}$ & $\delta B D=\sqrt{2}(\Delta x \alpha+\Delta y) \delta \alpha$ & $0.57 \cdot n(s)$ \\
\hline$\delta \Delta \mathrm{x}$ & Longitudinal jitter & $0.28 \cdot n(s) \mathrm{pm} / \sqrt{\mathrm{Hz}}$ & $\delta B D=\sqrt{2}\left(1+0.5 \alpha^{2}\right) \delta \Delta x$ & $0.40 \cdot n(s)$ \\
\hline$\delta \mathrm{a}$ & $\begin{array}{c}\text { Rotation axis } \\
\text { longitudinal jitter }\end{array}$ & $0.30 \cdot n(s) \mathrm{pm} / \sqrt{\mathrm{Hz}}$ & $\delta B D=\sqrt{2} \delta \alpha$ & $0.43 \cdot n(s)$ \\
\hline Total & & & & $1.40 \cdot n(s)$ \\
\hline
\end{tabular}

\subsection{Mechanical design}

The rotation of the mirror is guided by a so-called Haberland hinge, a monolithical elastic cross hinge. Due to the limitations on magnetic materials and contamination, magnetic, hydrostatic or contact bearings are not favored. The axis of rotation of the Haberland hinge is aligned with the mirror surface.

The material of choice is $\mathrm{Ti}_{6} \mathrm{Al}_{4} \mathrm{~V}$, due to its high allowable stress and high dimensional stability. The mechanical design is shown in Figure 2. The entire mechanism, excluding the functional components, is wire-eroded in a single fixture configuration; such that optimal production tolerances are achieved.

A compact elastic transmission between the actuator and mirror allows actuation of the mirror angle without introducing parasitic forces. The elastic transmission also enables the inclusion of a second, independent actuator, such that the mechanism is redundant. The required mechanical stroke of $\pm 412 \mu \mathrm{rad}$ can be actuated with an actuator stroke of 20 $\mu \mathrm{m}$, with minimal hysteresis effects.

To minimize OPD due to temperature variations, the thermal center of the $\mathrm{Ti}_{6} \mathrm{Al}_{4} \mathrm{~V}$ structure is placed in line with the axis of mirror rotation. This is achieved by strategic placement of isostatic interfaces to the optical bench, as shown in Figure 4. 


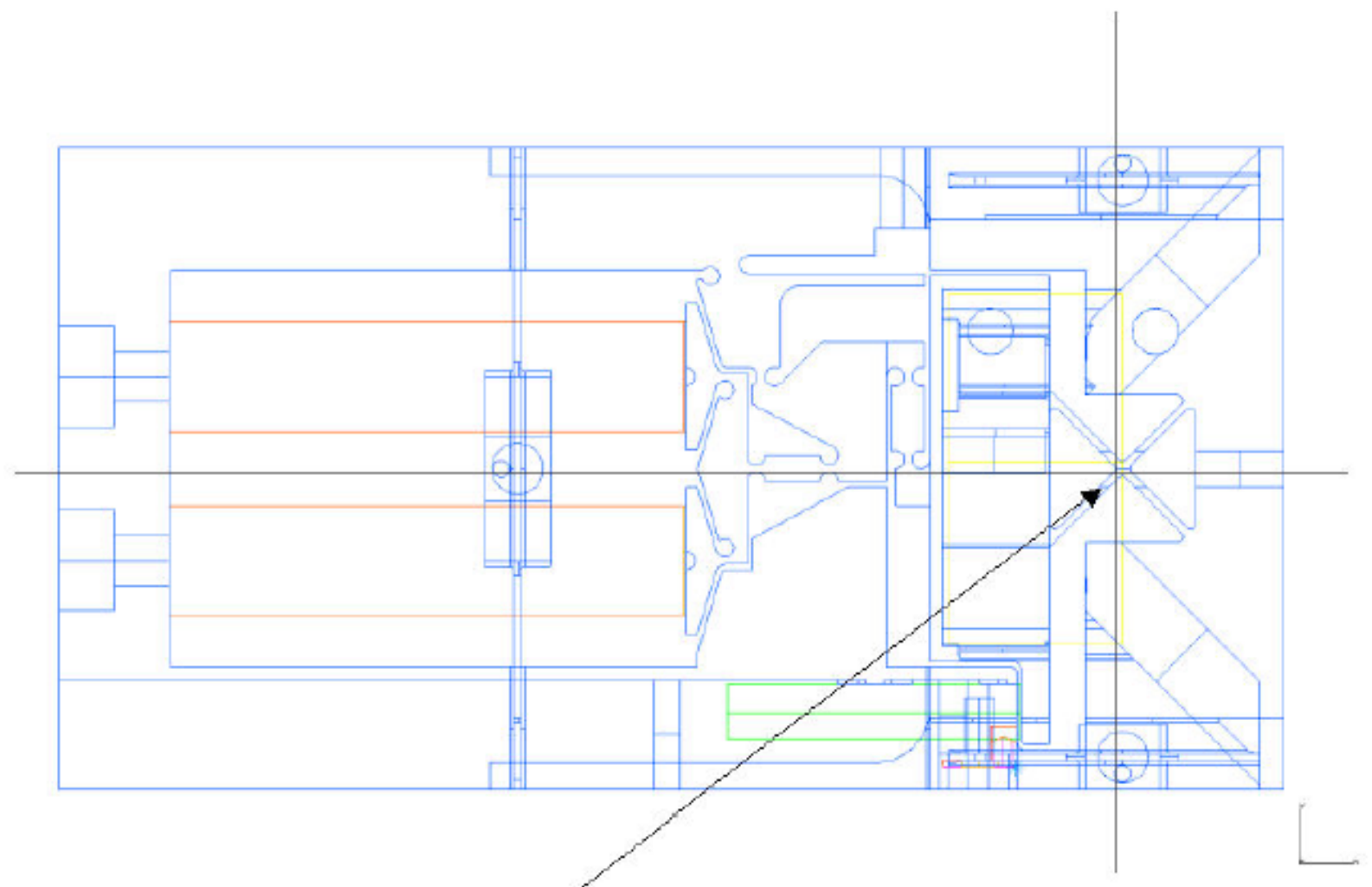

\section{Thermal centre of structure}

Figure 4: CAD drawing of the design of the PAAM. The intersection of the black lines indicates the thermal center of the mechanism, which has been designed to be on the axis of rotation.

A finite-element model analysis (FEM) of the Haberland hinge was used to predict the longitudinal jitter, caused by the coupling between angular jitter and piston sensitivity. Production tolerances of $\pm 10 \mu \mathrm{m}$ were included, which was justified by the accuracy and symmetry of the applied wire erosion process to manufacture the monolithical mechanism. Figure 5 shows the FEM model of the hinge in an extreme angle. Figure 6 shows the calculated piston movement of the mirror rotation axis under different actuation angles. The derivative of this curve is the piston sensitivity to angular jitter, which is $2.1 \mathrm{fm} / \mathrm{nrad}$ at the maximum angle. With the requirement of $8 \mathrm{nrad} / \sqrt{\mathrm{Hz}}$, the predicted longitudinal jitter becomes $0.017 \mathrm{pm} / \sqrt{\mathrm{Hz}}$, which is significantly below the allocated budget of $0.28 \mathrm{pm} / \sqrt{\mathrm{Hz}}$. 

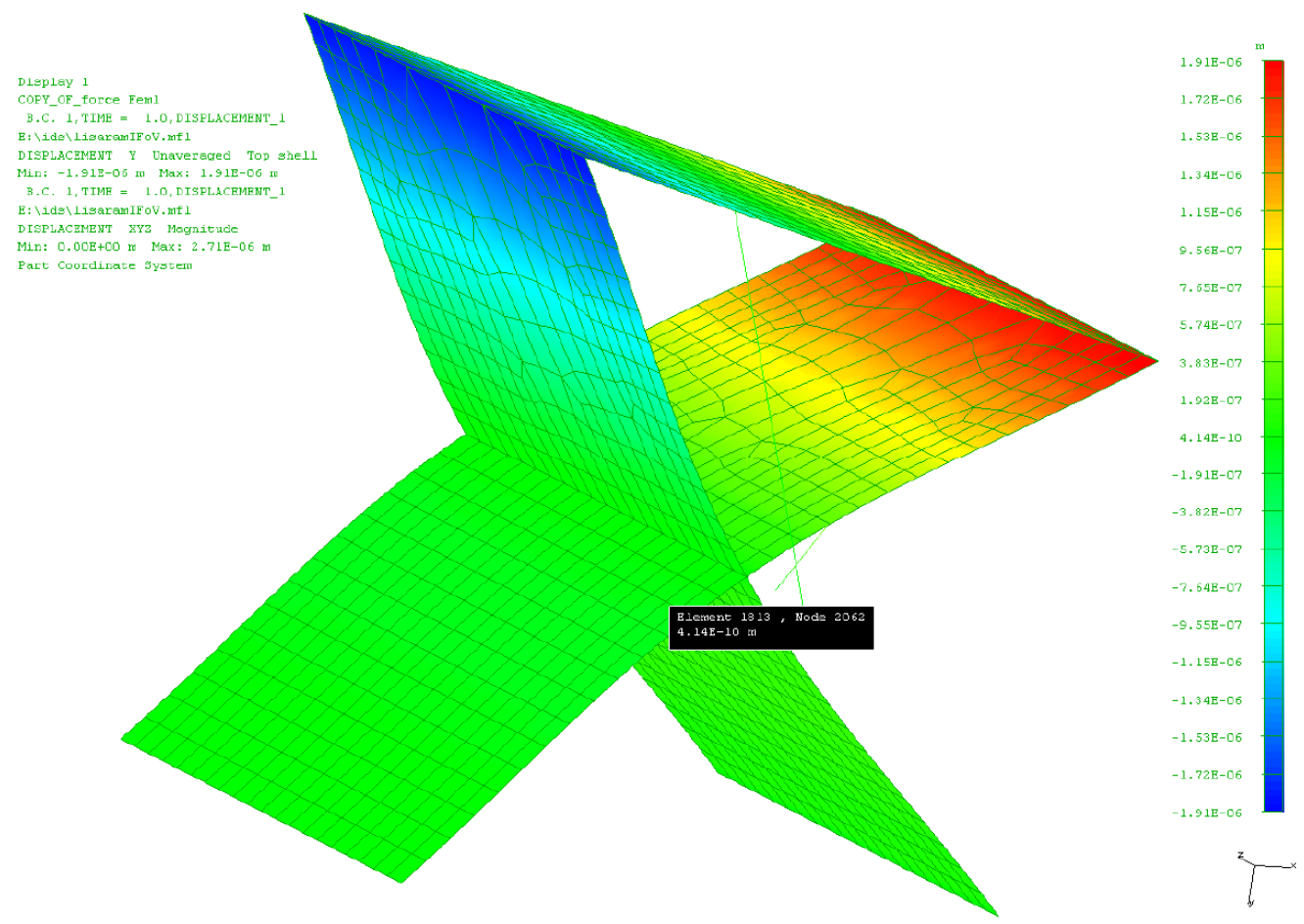

Figure 5: Meshed FEM model of the Haberland hinge, actuated to an extreme angle. Outcome of the FEM analysis is the longitudinal and lateral jitter of the axis of rotation. Production tolerances of $\pm 10 \mu \mathrm{m}$ are taken into account.

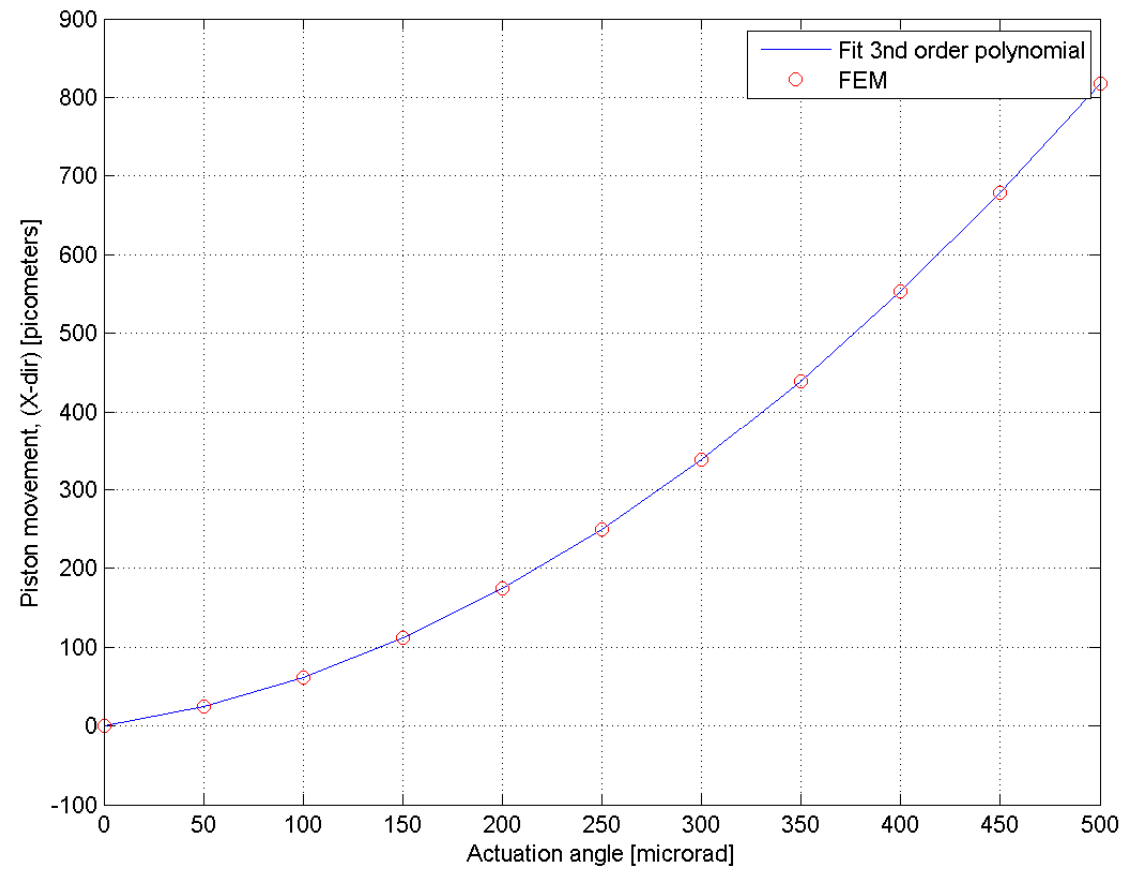

Figure 6: The piston movement of the axis of rotation under different actuation angles. By taking the derivative of the fitted polynomial, the piston sensitivity can be determined for different angles. 


\subsection{Electrical design}

For the actuation, two piezo stacks (PPA20M) of Cedrat Technologies are used. For the stroke of $20 \mu \mathrm{m}$, and with the limitations on magnetic materials, a piezo stack is the most appropriate actuator. The stacks are driven with a high voltage piezo amplifier (Cedrat Technologies LA75B), which produces voltages between -20 and $+150 \mathrm{~V}$.

Because of piezo hysteresis and discharge behaviour, a closed loop system is required to meet the requirement for angular jitter in the entire range. The controller acting between the sensor and piezo actuator can have a low bandwidth, because only disturbances within the LISA measurement band width have to be suppressed.

The sensor consists of an active target capacitive sensor system, which measures the displacement of the far end of the mirror (see Figure 7). The tilting of the mirror is less than $0.5 \mathrm{mrad}$, which has only a small influence on the capacitive sensor signal. A one-time calibration ensures that the sensor signal is representative of the mirror angle with high enough accuracy. The active target system is preferred over a passive target system for the following reason: in a passive target system, the cable capacitance is in parallel to the capacitance to be measured, and is typically a few orders higher. This makes it very sensitive to low frequency environmental changes which dramatically influence the cable capacitance. In an active target system, the target is connected to a virtual ground, which draws the current from the cable capacitance. This way, the system will be much less sensitive to environmental changes.

The capacitive probe is a standard Lion Precision probe, whereas the active target consists of an isolating BK7 plate, coated with a layer of gold. The active target, located on the moving part, is connected to the sensor cable by two thin copper wires, which add negligible parasitic stiffness to the Haberland Hinge. The capacitance-to-digital converter (CDC) electronics were chosen to be a custom electronics board, especially for extreme requirements on low frequency noise. Typically, capacitive sensor electronics introduce $1 / \mathrm{f}$ type noise in the amplification, but the charge-integration configuration and precision electrical components chosen for the CDC board reduced this effect maximally.

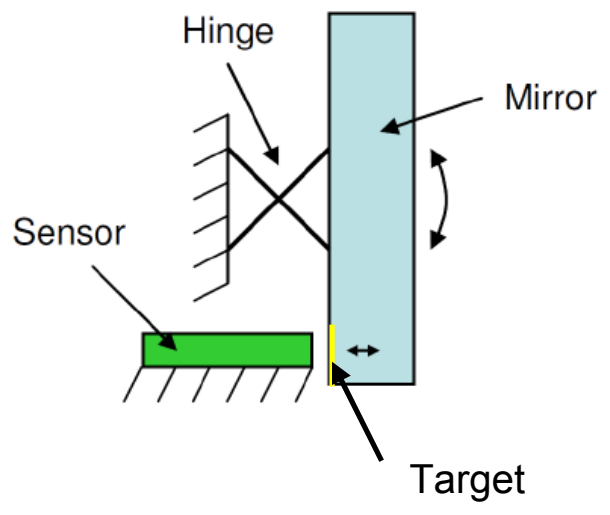

Figure 7: Schematic illustration of the active target capacitive sensor. The sensor measures the displacement of the tip of the mirror. The tilting of the mirror is taken into account by one-time calibration.

\subsection{Sensor noise testing}

Due to the closed loop operation of the PAAM and the low frequency performance requirements, the most critical noise source in the loop is the capacitive sensor. To test the performance of the CDC board in combination with the active target capacitance, a test set-up was designed to measure the noise. A stable reference capacitance was placed in a temperature $( \pm 1 \mathrm{~K})$ and humidity controlled environment, while the CDC board was kept in a normal laboratory environment. The resulting contribution of the electrical noise to the angular jitter in terms of amplitude spectral density is shown in Figure 8, calculated from the measurement using a simple model of the plant and a controller with a band width of approximately $10 \mathrm{~Hz}$. In worst case, the capacitive sensor noise is low enough to achieve the required performance. Especially the low magnitude of $1 / \mathrm{f}$ type noise is a remarkable performance achievement for a displacement sensor. Noise measurements of other electrical components in the control loop resulted in noise levels that were much lower than the capacitive sensor noise within the band width of interest. It is therefore justified to consider the noise level of the capacitive sensor as a total performance prediction. 


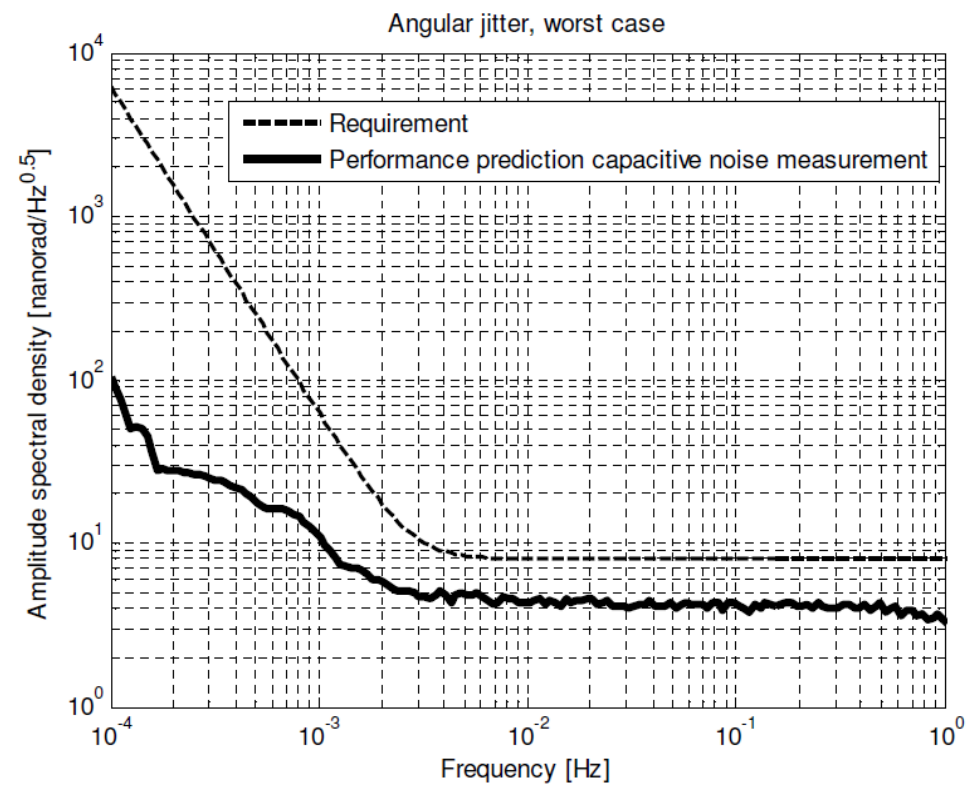

Figure 8: Performance prediction of the angular jitter, based on capacitive noise measurements under controlled environmental conditions. A dynamical model of the PAAM and the controller is included. The predicted performance is below specification.

\section{ENVIRONMENTAL AND PERFORMANCE TESTING}

\subsection{Environmental testing}

The specification for the launch loads is $25 \mathrm{~g}$ RMS. This random vibration load is applied to the Elegant Bread Board realization of the PAAM. The spectrum containing $25 \mathrm{~g}$ RMS was notched because of a lower Q-factor of the mechanism. In three translational degrees of freedom, an effective spectrum of approximately $20 \mathrm{~g}$ RMS was applied by a shaker. The response, measured with tri-axial accelerometers, showed no significant shift of resonance frequencies. After testing, visual inspection of the mechanism showed no degradation.

\subsection{Performance testing}

The performance of the Elegant Bread Board realization of the PAAM was measured with the help of the Albert Einstein Institute in Hannover, because of their experience with measuring picometer stabilities and the availability of an existing measurement facility ${ }^{2}$.

Figure 9 shows the test set-up used for the performance measurements, featuring a vacuum chamber pumped down to $10^{-4}$ mbar that is temperature controlled to less than $14 \mu K / \sqrt{H z} \cdot n(f)$. The extreme stability required to measure 1.4 $\mathrm{pm} / \sqrt{\mathrm{Hz}}$ can currently only be achieved with an interferometer, realized with a resonance cavity. Because the nominal incidence angle of the PAAM is 45 degrees, the cavity is made triangular. To keep the cavity resonant, the stabilized laser frequency that is generated in an external cavity is modulated by a controller. The actuator is an Electro-Optical Modulator, which is included in the vacuum chamber. The sensor is a frequency counter, which measures the resonance frequency of the cavity relative to the reference cavity. The feedback signal is a measure of the cavity round trip length change, which represents the contribution of the mechanism to the OPD. The cavity of the measurement set-up inside the vacuum chamber is mounted on a Zerodur base plate, to minimize the influence temperature variations on the cavity round trip length. 


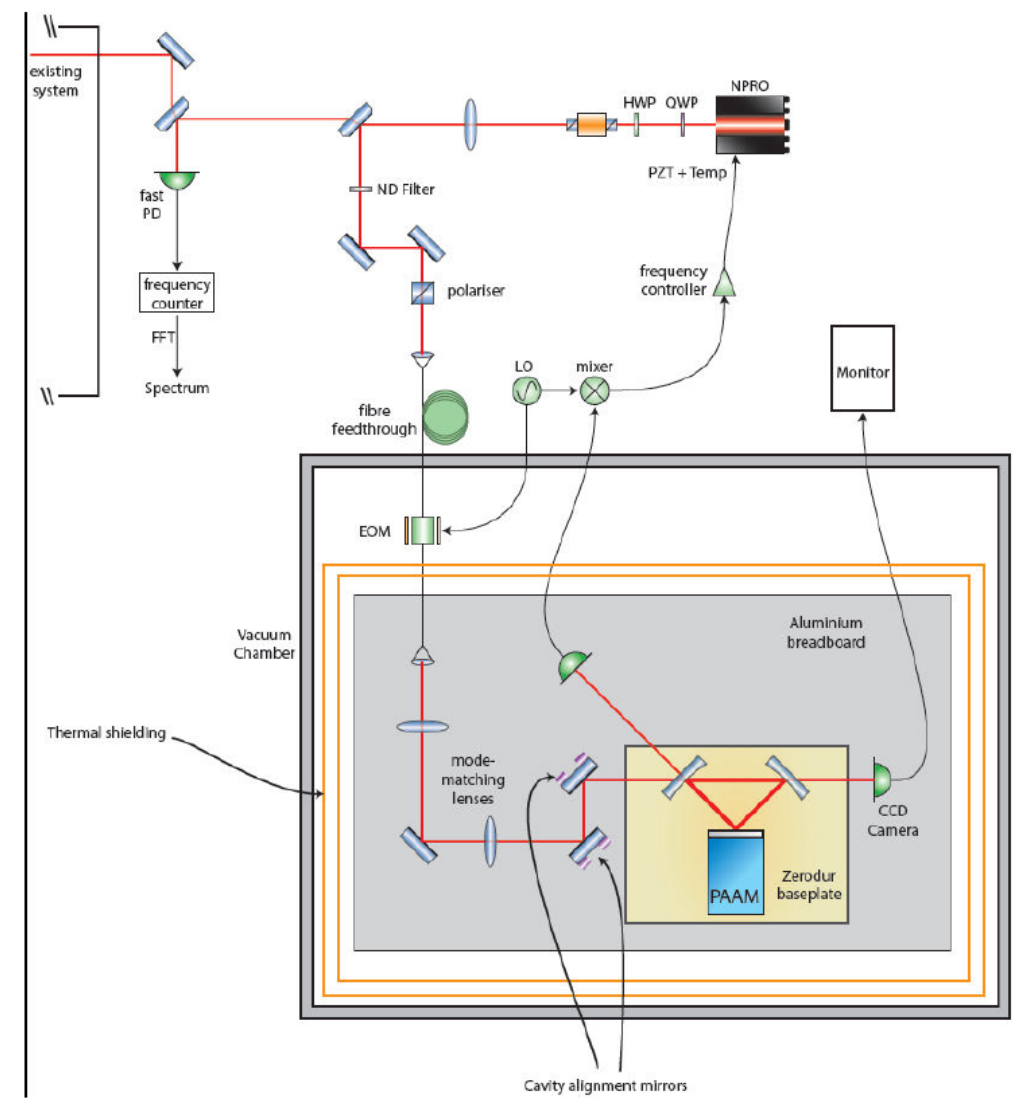

Figure 9: Figure courtesy of Albert Einstein Institute, Hannover. The OPD of the PAAM is measured in a triangular resonance cavity, inside a temperature controlled vacuum tank.

\subsection{Test description}

Two of the critical requirements of the PAAM need to be verified by tests: the OPD and the angular jitter. Both are measured at three different static angles within the range of the mechanism. The LISA measurement band width determines the length of each performance measurement: the lowest frequency to be measured is $0.03 \mathrm{mHz}$. With several repetitions of this period to obtain a proper spectral estimate, the measurement time per angle is approximately 48 hours.

To measure the OPD, the PAAM has to be aligned accurately in the resonance cavity. To achieve this, a modulation sine wave is used as a set point on the mechanism, which will follow this signal by rotation of the mirror. Through the amplitude of the sine wave, the misalignment of the axis of rotation with respect to the incoming beam can be estimated.

To measure the angular jitter, the PAAM is deliberately placed at an alignment offset. A modulation sine wave is used as a set point for the PAAM. Through the amplitude of the sine wave, the coupling of angular jitter to OPD can be estimated. A long measurement of OPD will then provide an indirect measurement of the angular jitter of the PAAM.

\subsection{Test performance results}

The results of the performance measurements of OPD and angular jitter are shown in Figure 10 and Figure 11. For all angles, the performance of the mechanism is exactly according to the requirements. The peak visible at $50 \mathrm{mHz}$ is the modulation sine wave which is used for alignment in the OPD measurements, and coupling estimation in the angular jitter measurements. 


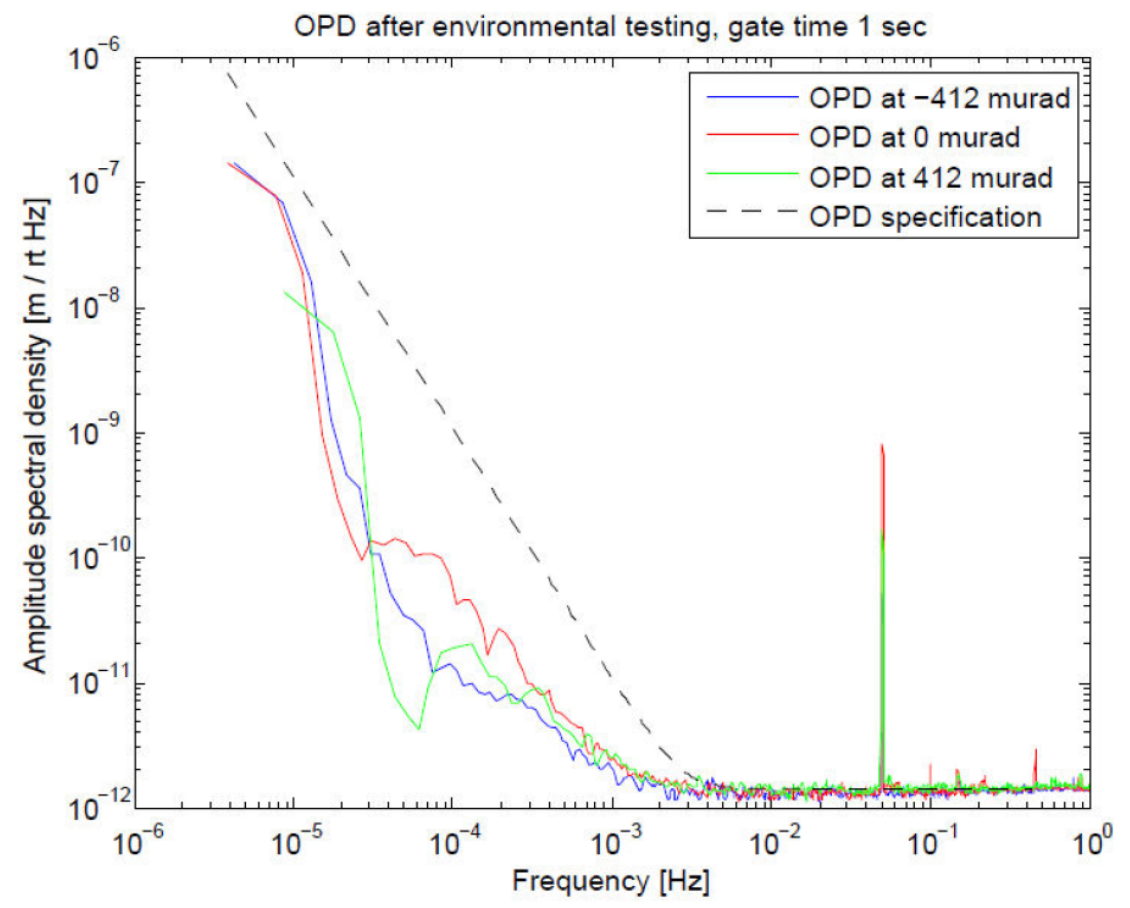

Figure 10: Amplitude Spectral Density of the OPD measurements on the PAAM. For all angles, the result meets the requirements. The peak at $50 \mathrm{mHz}$ is introduced for alignment in the cavity.

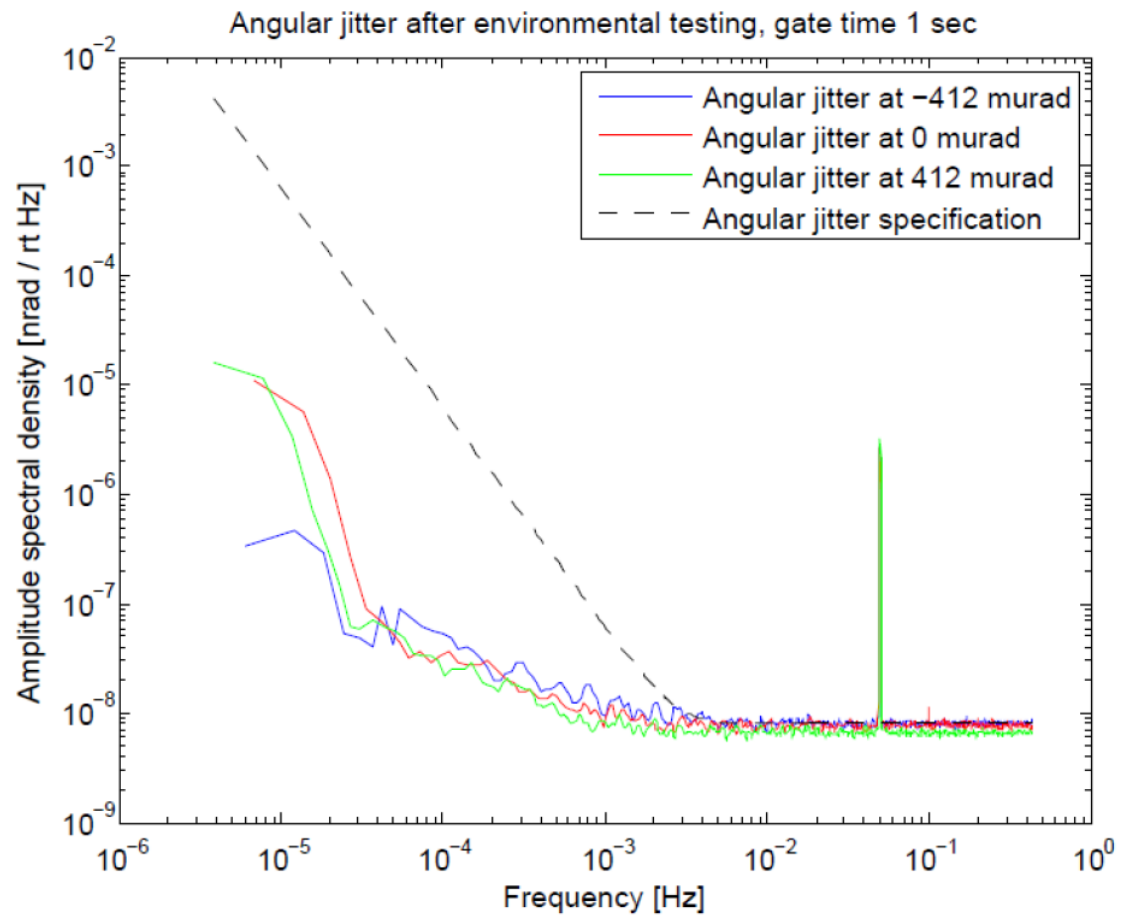

Figure 11: Amplitude Spectral Density of the angular jitter measurements on the PAAM. The PAAM is deliberately placed at and alignment offset, to make the angular jitter dominant in the OPD measurement. For all angles, the result meets the requirements. The peak at $50 \mathrm{mHz}$ is introduced to estimate the coupling of angular jitter to OPD. 


\section{CONCLUSION}

With the design, realization and testing of the PAAM, TNO Science \& Industry has demonstrated that a scanning mechanism with picometer stability and under extreme environmental conditions is achievable. Performance test measurements have shown that it is compliant with the challenging requirements for optical path delay and angular jitter.

The analysis, as well as the measurement results, shows that application of a Haberland hinge in a monolithical structure enables rotation with negligible parasitical motion in terms of OPD. The thermal design of the structure showed to be sufficient to guarantee the thermal stability.

The angular jitter measurements have shown that an active target capacitive sensor system meets the extreme requirements on low frequency noise.

The Elegant Bread Board of the PAAM, as used in the performance testing, will be included in a bread board model of the LISA Optical Bench in the near future.

\section{REFERENCES}

[1] Danzmann, K., "LISA Mission Overview”, Advances in Space Research Vol. 25, Issue 6, 2000, 1129 - 1136

[2] Troebs, M., "Laser development and stabilization for the spaceborne interferometric gravitational wave detector LISA", PhD Dissertation, 2005. 